\title{
Formation of current density profile in tilted current sheets
}

\author{
A. A. Petrukovich ${ }^{1}$, W. Baumjohann ${ }^{2}$, R. Nakamura ${ }^{2}$, and A. Runov, ${ }^{2, *}$ \\ ${ }^{1}$ Space Research Institute, 84/32 Profsoyuznaya st., Moscow, 117997, Russia \\ ${ }^{2}$ Space Research Institute, Austrian Academy of Sciences, Schmiedlstr. 6, 8042 Graz, Austria \\ *now at: Institute of Geophysics and Planetary Physics, UCLA, Los Angeles, CA-90095, USA
}

Received: 2 April 2008 - Revised: 7 October 2008 - Accepted: 7 October 2008 - Published: 20 November 2008

\begin{abstract}
We investigate Cluster observations of strongly tilted sheets (flapping events) in the magnetotail. In accordance with the simple model of slip deformation (vertical differential displacement of neighboring flux tubes), the $J_{y}$ current density component in the tilted sheet remains constant and equal to that in the horizontal undisturbed sheet. However, a substantial $J_{z}$ component appears proportional to the local sheet tilt. Slip-type variations, having smaller scale than the full crossing, locally change the tilt and $J_{z}$ and may thus create a variety of non-classical (bifurcated, asymmetric etc) current density profiles.
\end{abstract}

Keywords. Magnetospheric physics (Magnetotail; Plasma sheet)

\section{Introduction}

In the magnetotail relatively fast (some hundreds of seconds) large-amplitude (tens of nT) variations of $B_{x}$ are easily noticeable and are usually attributed to flapping sheet motions. They also provide a convenient instrument for current sheet investigations. The first four years of Cluster observations revealed structural complexity of the plasma sheet (e.g. Sergeev et al., 2003, 2004; Runov et al., 2005a). In particular, significantly inclined current sheets are abundant and alternating tilts are often observed within minutes, suggesting a meso-scale structure rather than large-scale flapping. The current sheet thickness during flappings was often from several hundreds to a few thousands km, even when an expanded sheet was nominally expected (e.g., associated with northward IMF and near-flank crossings with large $B_{z}$ ).

Besides current sheet tilts, Cluster can determine current density and, assuming some stationarity, its spatial profile along the normal. A variety of embedded, bifurcated and

Correspondence to: A. A. Petrukovich

(apetruko@iki.rssi.ru) asymmetric sheet shapes were discovered, while the classical Harris profile was in a minority (Asano et al., 2005; Runov et al., 2006, 2003; Sergeev et al., 2003; Petrukovich et al., 2007).

Interpretation of such events may differ depending on the observed magnetic field geometry, the local plasma conditions, as well as the magnetospheric configuration. In several targeted investigations, some such events were interpreted as a wavy displacement of the main cross-tail current sheet plane, propagating flankwards (Zhang et al., 2002), or as a quasi-stationary structure of vertically shifted flux tubes, flapping azimuthally around the spacecraft location (Petrukovich et al., 2003). Some observations of bifurcated sheets were associated with x-line geometry (Runov et al., 2003; Thompson et al., 2006).

Petrukovich et al. (2006) considered the cross-tail current sheet formed by curved magnetic flux tubes as a threedimensional object, in which two types of meso-scale deformations may take place (Fig. 1). During a bend-type deformation the flux tubes rotate and follow the sheet normal direction. Alternatively, during a slip-type deformation, the flux tubes shift vertically relative to their neighbors, but the magnetic field orientation remains the same, irrespective of the normal direction and the sheet tilt. Quiet-time stronglytilted sheets, forming a significant subset of Cluster flapping events, were consistent with a slip-type displacement (see also Sharma et al., 2008). A good example is the 3 August 2004 event (Fig. 2, adapted after Petrukovich et al., 2006). Even rather small $\sim 5 \mathrm{nT}$ variations in the $B_{x}$ curve (panel b) have leading and trailing edges with different tilts (panel d). However $B_{z}$ (panel c) is large and almost constant. $B_{y}$ is much smaller than $B_{z}$ (not shown here, see Petrukovich et al., 2006, for details). Current density profiles for some crossings of this event are rather different, including asymmetric (curves 1,2) and bifurcated (curve 3) forms (panel a). Such diversity of neighboring crossings is rather typical for Cluster observations.

Published by Copernicus Publications on behalf of the European Geosciences Union. 


\section{Flappings: magnetic configuration}

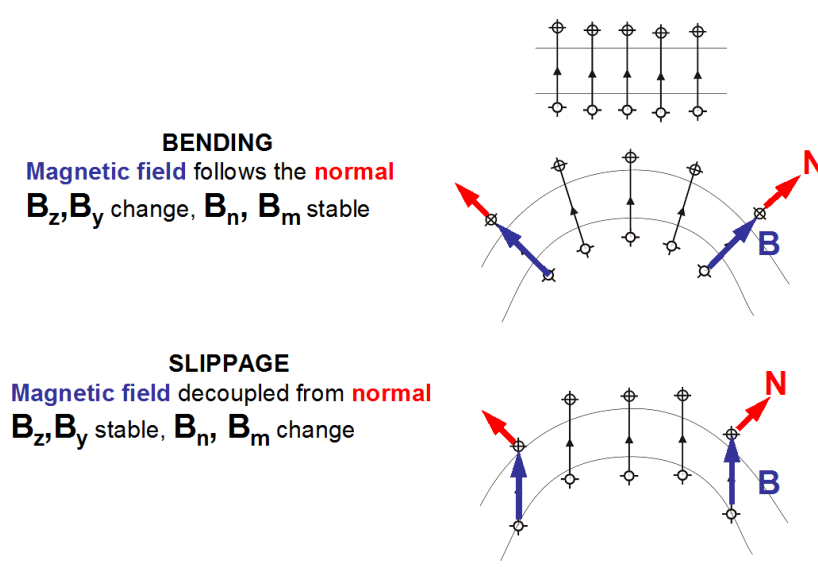

Fig. 1. Two variants of the cross-tail current sheet deformation, shown in a sunward view in $Y Z$ GSM plane, with $B_{y}=0$. See text for details. Modified from Petrukovich et al. (2006).

While Petrukovich et al. (2006) analyzed tilts and the magnetic configuration of the sheets, in this investigation we further model current density profiles during slip-type deformations, and compare them with observational data. We also describe relevant data processing approaches, since interpretation of current density profiles turns to be rather sensitive to proper selection of coordinate frames.

\section{Techniques of current sheet description}

Cluster magnetic data were used in our experimental analysis (Balogh et al., 2001). Hereafter components $x, y, z$ are in the GSM frame of reference. $l, m, n$ are in the proper frame of an idealized planar sheet: maximum variance direction, electric current direction, normal direction. The angles $\phi, \theta$ (of the normal direction) are in a polar coordinate system, with $X$ GSM as the polar axis. The polar angle $\theta$ (latitude) is measured from the $Y Z$ plane, positive - Earthward (it will not be used in this investigation). The azimuthal angle $\phi$ (in the $Y Z$ plane) is measured from the $Z$ axis (positive for normals with positive $Y$ component). In such a frame zero angles correspond to a horizontal sheet with the normal along $Z$ GSM, while the azimuthal angle describes the most commonly observed tilts within the $Y Z$ plane (Sergeev et al., 2004).

In the magnetotail plasma sheet 4-point magnetic gradients are usually interpreted in the approximation of a uniform planar current sheet. In the idealized sheet the normal is equivalent to the $B_{x}$ gradient direction. However, if the actual magnetic maximum variance direction is orthogonal to $X$, it might be necessary to use gradient of the maximum variance component $B_{l}$. Another method is to determine normals, analyzing inter-spacecraft time delays within the crossing (equivalent to computation of "time" gradient $\Delta t / \Delta r$ at some fixed magnetic field value (Runov et al., 2005b)). Gra- dients are assumed to be constant on the scale of spacecraft separation, otherwise errors in the normal determination will appear. In particular, if the current sheet thickness is comparable with the spacecraft separation, the computed gradients will be smaller than the real ones (Runov et al., 2005b).

Other sheet characteristics are the maximum variance direction, defining the main sheet magnetic component $B_{l}$, and the electric current direction (computed as $\nabla \times B$ ). For many flapping events the timing and magnetic gradient normals were coincident and orthogonal to the maximum variance and the current directions with an accuracy of about $10^{\circ}-20^{\circ}$. Therefore the planar sheet approximation appears to be generally acceptable. Since the angles between the experimentally determined normal, maximum variance and shear directions are not exactly $90^{\circ}$, a similar orthogonal proper frame of reference is usually established. In our investigations the algorithm was: $l-$ along maximal variance, $m=n^{*} \times l\left(n^{*}\right.$ is magnetic or time normal), $n=l \times m$. Note that, since the final $l m n$ system is established once for a single crossing, any variations overlaying the overall structure are averaged out. Magnetic gradient normal and electric current density vectors are available with the time resolution of magnetic field measurements.

Establishment of the proper frame of reference is a key step towards analysis of the sheet structure (in particular, identification of the Harris-type current density profile or any deviations from it). A single 4-point measurement is sufficient to determine the linear change (gradient vector) only. Still, some estimates can be done, involving extra physical arguments. For a given normal (gradient) direction, one can estimate the degree of non-linearity, e.g. comparing differences in pairs of satellites. Asano et al. (2005) thus suggested that a majority of sheets are embedded, while Harris sheets are a clear minority.

When the current sheet moves past the spacecraft, a time sequence of measurements $B(t)$ and $J(t)$ can be converted to spatial profiles (Runov et al., 2005a). This method assumes stationarity (in particular, fixing a single $l m n$ coordinate frame). Variations of current density along the normal are then interpreted as a true profile of a sheet. Results are more confident in fortuitous occasions when the spacecraft is moving back and forth several times across a current sheet, revealing the same structure relative to the local magnetic field (e.g. Runov et al., 2003; Sergeev et al., 2003; Petrukovich et al., 2007).

\section{The model}

We describe a slip-type deformation (see Sect. 1 for the definition) of a planar horizontal thick current sheet with the following simple model. The sheet magnetic field originally depends only on the $Z$ coordinate $B=B_{x}=B_{l}=B_{0} \tanh \left(\left(z+z_{0}\right) / H_{z}\right), \quad B_{y}=0, \quad B_{z}=$ const, the current $J$ is along Y-axis. Each curved flux tube $\left(B_{x}, B_{z}\right)$ 

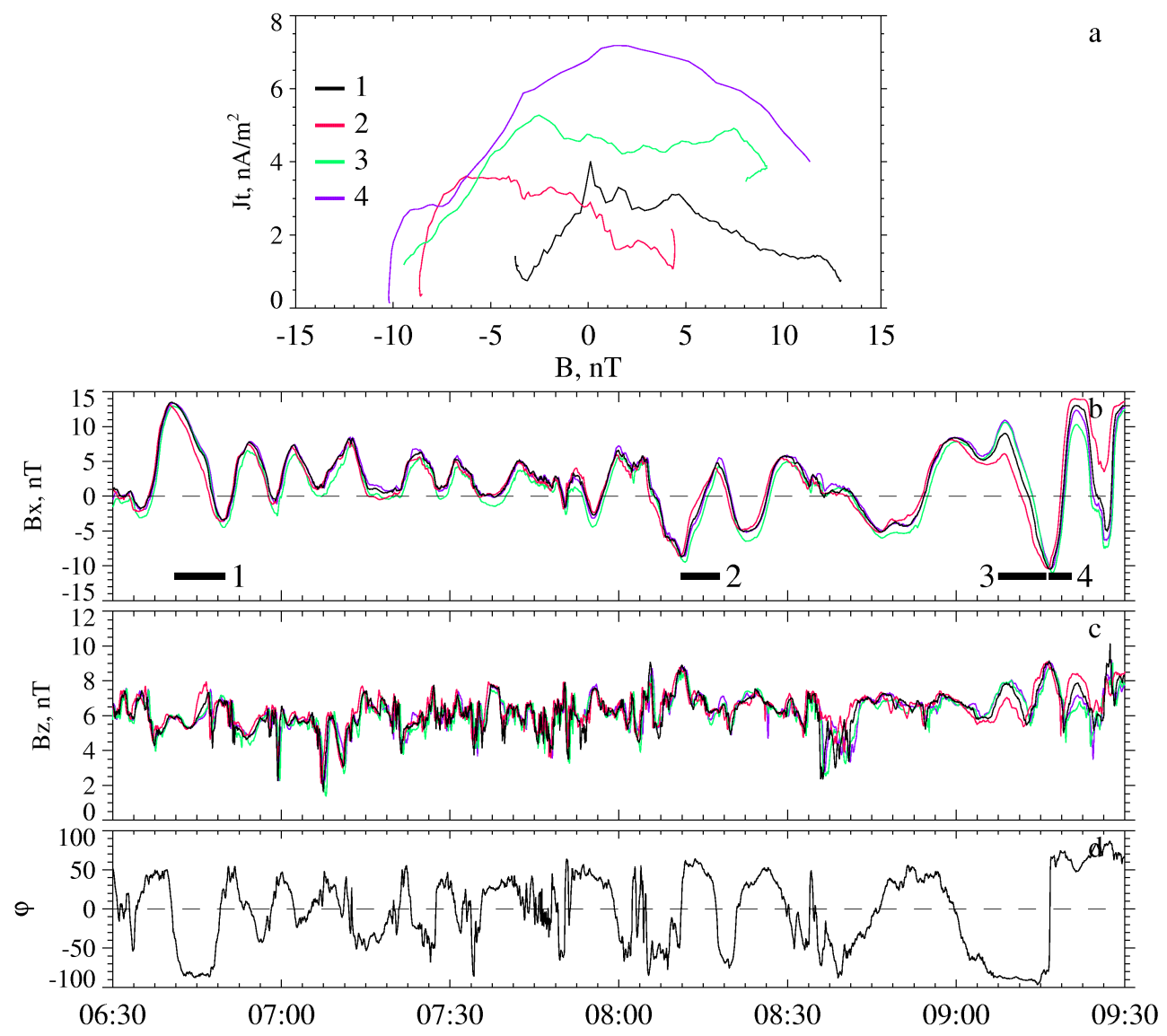

Fig. 2. The event of 3 August 2004: (a) Current density profiles across the sheet. Magnetic field $B_{x}$ (b), $B_{z}$ (c) in GSM frame of reference and normal direction angle (d) relative to Z GSM (see Sect. 2 for details). C1,C2,C3,C4 spacecraft are denoted by standard colors black,red,green,blue, respectively. Thick horizontal black lines in panel (b) denote the intervals of the profiles in (a). Modified from Petrukovich et al. (2006).

is characterized by its position $y$. Then the flux tubes are shifted vertically so that $z_{0}=z_{0}(y)$.

For a clearer understanding we start with a single step-like function with $B_{0}=25 \mathrm{nT}, H_{z}=10000 \mathrm{~km}$, $z_{0}=A_{z} \tanh \left(y / H_{y}\right), H_{y}=5780 \mathrm{~km}, A_{z}=10000 \mathrm{~km}$ (Fig. 3a). The maximum tilt at the origin is $\tan \left(\phi_{\max }\right)=A_{z} / H_{y}$ $\left(60^{\circ}\right.$ in Fig. 3a). Then $J_{y}=d B / d z=\cosh ^{-2}\left(\left(z+z_{0}\right) / H_{z}\right)$, $J_{z}=d B / d y=\cosh ^{-2}\left(\left(z+z_{0}\right) / H_{z}\right)\left(A_{z} / H_{y}\right) \cosh ^{-2}\left(y / H_{y}\right)$. $J_{y}$ remains constant and equal to its original Harris value in each moving flux tube irrespective to the tilt, but an additional non-Harris $J_{z}$ appears. When crossed by a spacecraft at the "center" of the step, such a configuration exhibits a higher and narrower total current density peak, than the initial horizontal (Harris) profile. Therefore a thin tilted sheet, embedded in the thicker original horizontal sheet, forms. Cases with larger tilts have larger $J_{t}=\sqrt{J_{x}^{2}+J_{y}^{2}}$ (Fig. 3b). If a crossing is at the "knee" of the kink (Fig. 3a), the maximum of the current density does not coincide with the minimum of the magnetic field (Fig. 3c), and asymmetric profiles appear (basically due to non-planarity of the configuration).

A more developed model is shown in Fig. 4. With three virtual spacecraft we observe a sheet with the wavy modification $z_{0}=A_{z} \cos \left(y / H_{y}\right)$. There is no dependence on $X$ and three spacecraft are enough for a gradient determination. One spacecraft is shifted from the base level by $1000 \mathrm{~km}$ in $Y$ (green curve), another by $1000 \mathrm{~km}$ in $Z$ (red curve). We select $B_{0}=30 \mathrm{nT}, A_{z}=7500 \mathrm{~km}, H_{z}=10000 \mathrm{~km}, H_{y}=2500 \mathrm{~km}$, and the maximum vertical velocity is $60 \mathrm{kms}^{-1}$. The spatial sequence is converted to a temporal sequence, assuming that the structure propagates along $Y$ with a velocity of $20 \mathrm{kms}^{-1}$, so that the period is of the order of $13 \mathrm{~min}$. The length of this simulation interval is $50000 \mathrm{~km}(45 \mathrm{~min})$. The maximum sheet tilt is $\phi_{\max }=\arctan \left(A_{z} / H_{y}\right) \sim 72^{\circ}$. In agreement with the previous example, $J_{z}$ changes sign and oscillates $\pm 6 \mathrm{nAm}^{-2}$ following the tilt, while $J_{y} \sim 2 \mathrm{nAm}^{-2}$ is almost constant (Fig. 4c). While the oscillation is sinusoidal, the tilt profile has more rectangular form, steeper for larger maximal tilts (not shown here). 


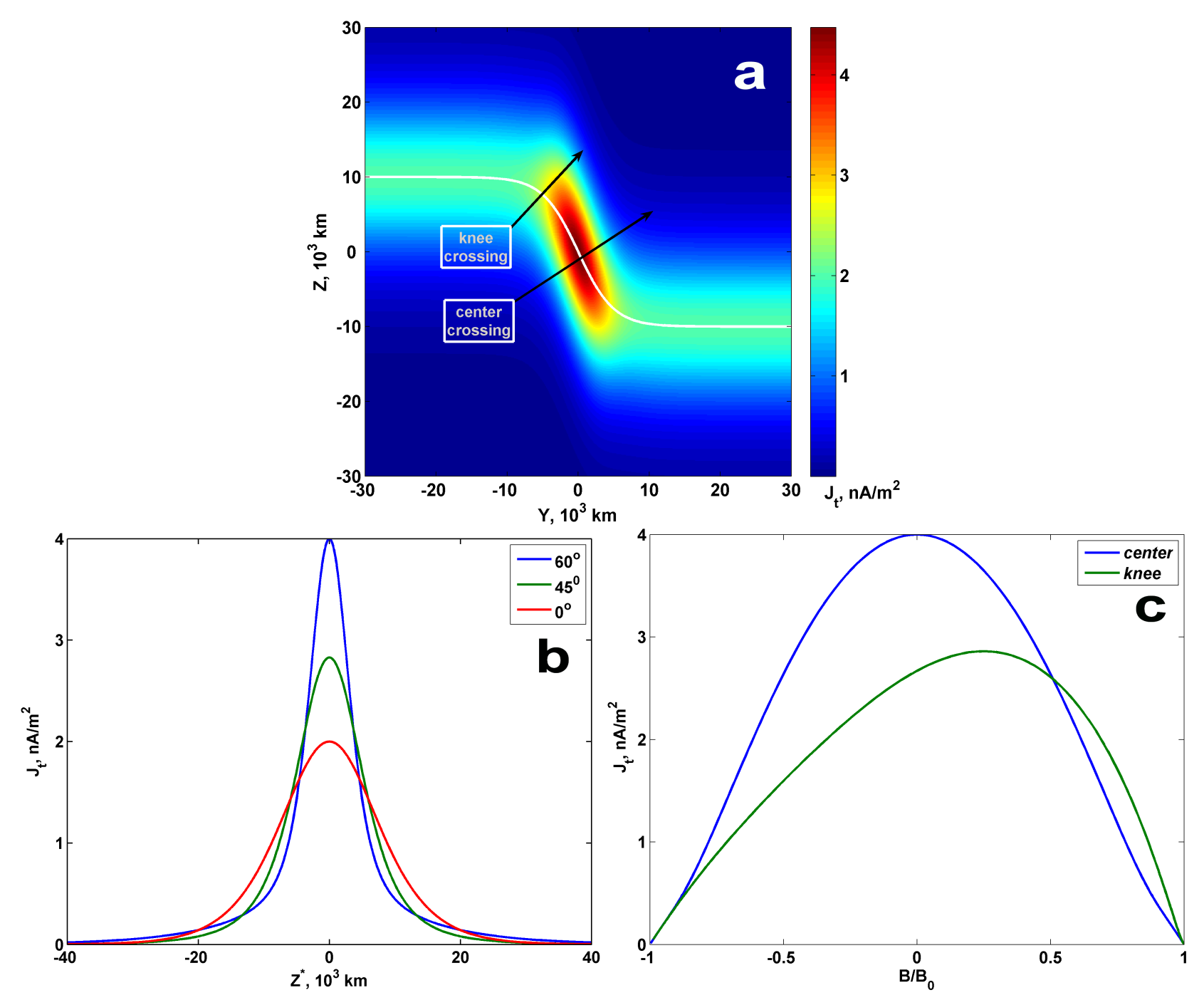

Fig. 3. The model kinked current sheet (see text for details). (a) Total current density map and the neutral sheet plane (white curve). Two trajectories of a virtual spacecraft denote a "center crossing" and a "knee crossing". (b) Total current density profiles relative to distance along the normal for a "center crossing" for different tilts of the sheet. (c) Nonsymmetric total current density profile relative to the local magnetic field during the "knee crossing", compared with the "center crossing" for the $60^{\circ}$ tilt model.

In the last example (Fig. 5) we add a second harmonic with a scale shorter by the factor of 3.62 (which was selected almost arbitrarily to keep the two periods substantially different), a maximum vertical velocity of $10 \mathrm{kms}^{-1}$ and an amplitude of $340 \mathrm{~km}$. The vertical amplitude of the main wave is reduced to $5000 \mathrm{~km}$ (maximum velocity $40 \mathrm{kms}^{-1}$ ). This addition results in a more realistic irregularity of the instantaneous tilt (Fig. 5d). Current density profiles taken at four major crossings (or flapping events in observational terms) are visually rather different, with asymmetric and bifurcated forms, because the second harmonic locally changes the tilt and $J_{z}$. The maximum current differs by a factor of 1.5 (Fig. 5a). Therefore, while the "complete" major crossings are well defined, being determined by the larger-scale wave, their inner structure varies substantially due to a local non-stationarity and inhomogeneity, caused by the second smaller-scale harmonic.

Finally we summarize the signatures of a model slip-type deformation in a thick Harris sheet: (1) the $J_{y}$ current density remains almost constant and equal to the nominal (Harris) value in the undisturbed sheet; (2) the $J_{z}$ current density varies substantially, following the tilt and may be substantially larger than $J_{y}$, the addition of $J_{z}$ creates a thin intensified embedded current sheet; (3) depending on the structure of the deformations (knees, multi-harmonic oscillations, etc) variations of $J_{z}$ may create complicated current density profiles with bifurcations, asymmetry etc. 

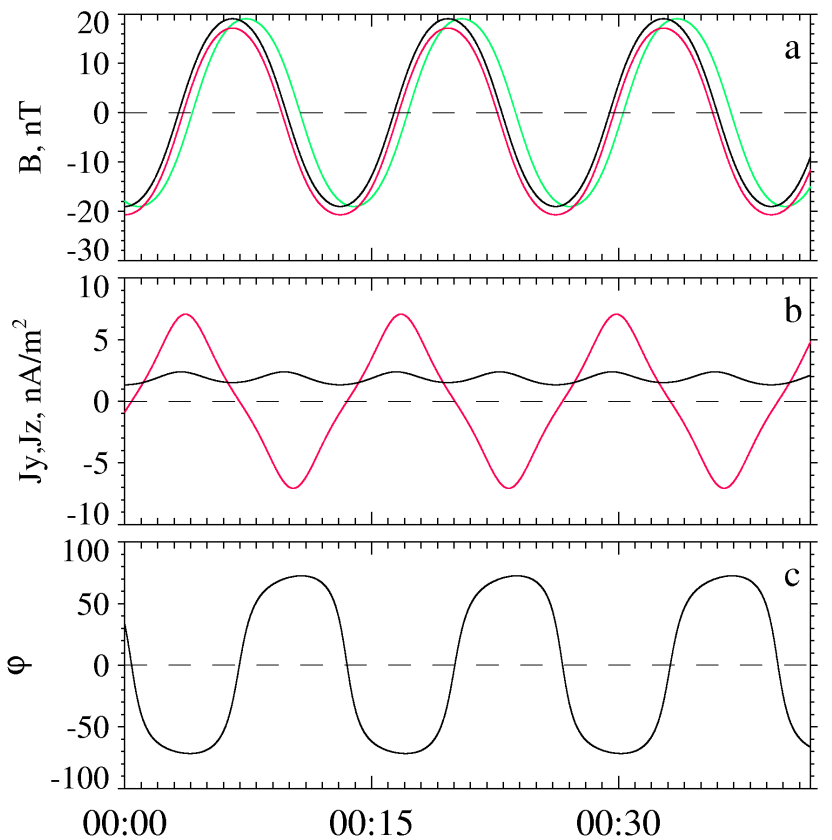

Fig. 4. Model current sheet with one monochromatic wave. (a) Magnetic field $B_{x}$. Colors denote three virtual satellites (see text for positions). (b) $J_{y}$ (black) and $J_{z}$ (red) current density. (c) $\phi$, tilt of the normal in the Y-Z plane. The time axis is in hh:mm format (arbitrary time).

\section{Comparison with observations}

We use events from several published earlier investigations to perform comparisons with our models. Figure 6 contains three crossings from the event of 3 August 2004 in the format of Fig. 4. In accordance with the model $J_{y}$ is about $1 \mathrm{nAm}^{-2}$ and $J_{z}$ varies through $\pm 2 \mathrm{nAm}^{-2}$ following the tilt. Smaller negative $J_{x}$ is likely a part (together with $J_{y}$ ) of the nominal cross-tail current, flowing more azimuthally. Its sign is consistent with the Cluster spacecraft location in the morning sector. Therefore the observed current density variations support the proposed slip deformation scheme.

The full set of individual crossings in the 3 August 2004 event have a wide range of tilt angles, and it is convenient to test the $J_{z}$ dependence on tilt in a more statistical way. $J_{x y}=\sqrt{J_{x}^{2}+J_{y}^{2}}$ and $J_{z}$ values were averaged over the middle of each crossing (Fig. 7). Indeed $J_{x y}$ is always stable and rather small $\left(\sim 1-2 \mathrm{nAm}^{-2}\right)$ in agreement with relatively thick background plasma sheet with large $B_{z}$. There is some regular increase of $J_{x y}$ towards positive tilts, probably related with small non-horizontality of the background sheet, not taken into account here. The sign and magnitude of $J_{z}$ depend on the tilt angle in agreement with the model.

The current density profiles taken at four observed crossings with the larger magnetic field span (Fig. 2a) exhibit factor of two changes of maximal total current density from case to case as well as asymmetric and bifurcated shapes

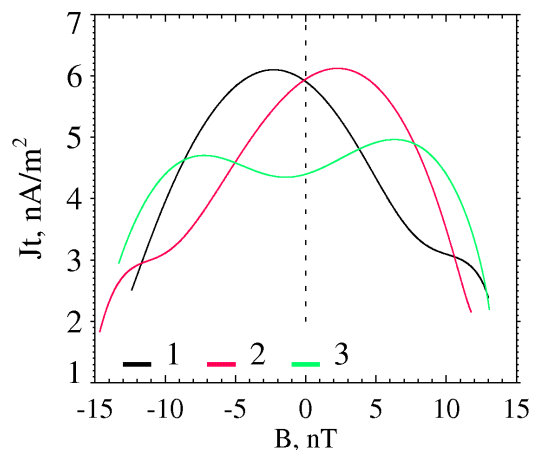

a

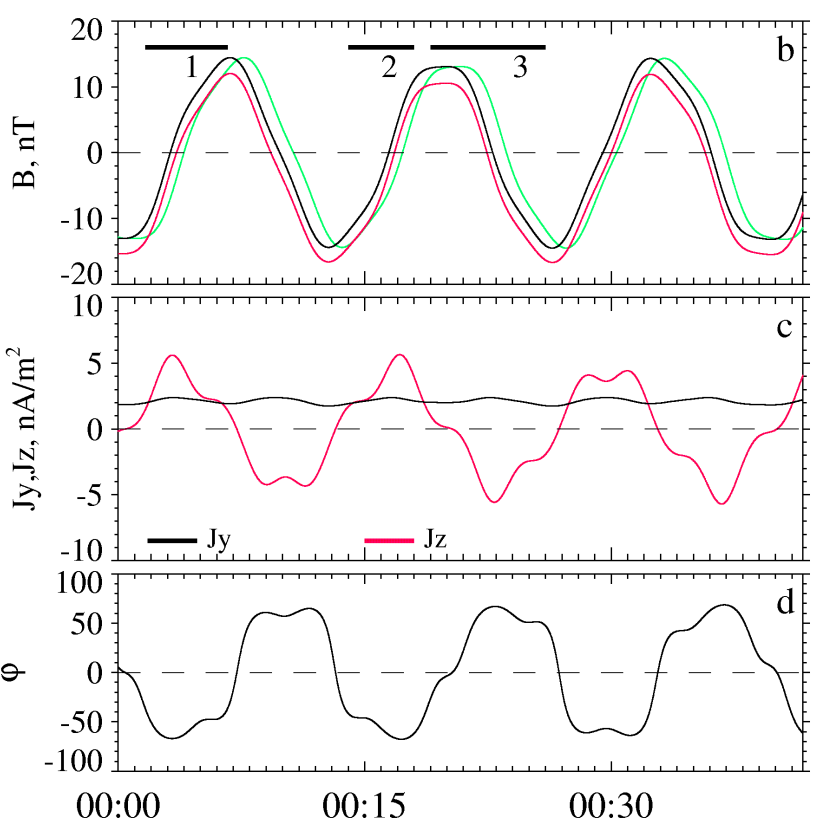

Fig. 5. Model current sheet with two monochromatic waves. (a) Current density profiles for three individual crossings. (b) Magnetic field $B_{x}$. Colors denote three virtual satellites (see text for positions). (c) $J_{y}$ (black) and $J_{z}$ (red) current density. (d) $\phi$, tilt of the normal in the $Y Z$ plane. The time axis is in hh:mm format (arbitrary time). Thick horizontal black lines in panel (b) denote the intervals of the profiles in (a).

with maxima at 5-10 nT. Similar shapes were reproduced also with our model (Fig. 5a). Especially noteworthy is consistency between the bifurcated profiles (\#3 in both figures), which was achieved without any special tuning of the model parameters. Although there is no close correspondence between Figs. $2 \mathrm{a}$ and $5 \mathrm{a}$ in all profiles, we used only two periodic oscillations, and with a more tuned model one could obtain almost any required profile.

Since the 3 August 2004 event is just a case study, it is reasonable to check predicted differences between $J_{y}$ and $J_{z}$ on a broader set of observations. Profiles of 16 nonhorizontal sheets (with $N_{y}>N_{z}$ ) taken from the set assembled by Runov et al. (2006) are averaged in Fig. 8. The $J_{z}$ profile appears to be narrower and more variable, while $J_{y}$ 


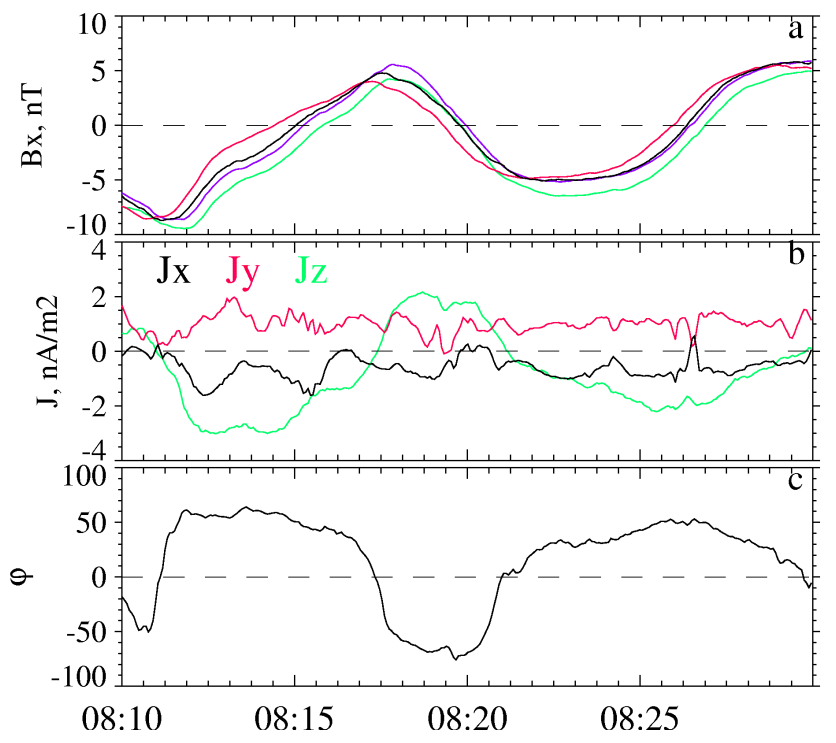

Fig. 6. Three crossings from the 3 August 2004 event in the format of Fig. 4. (a) $B_{x}$ magnetic field, (b) Components of current density, (c) $\phi$, tilt of the normal in the Y-Z plane.

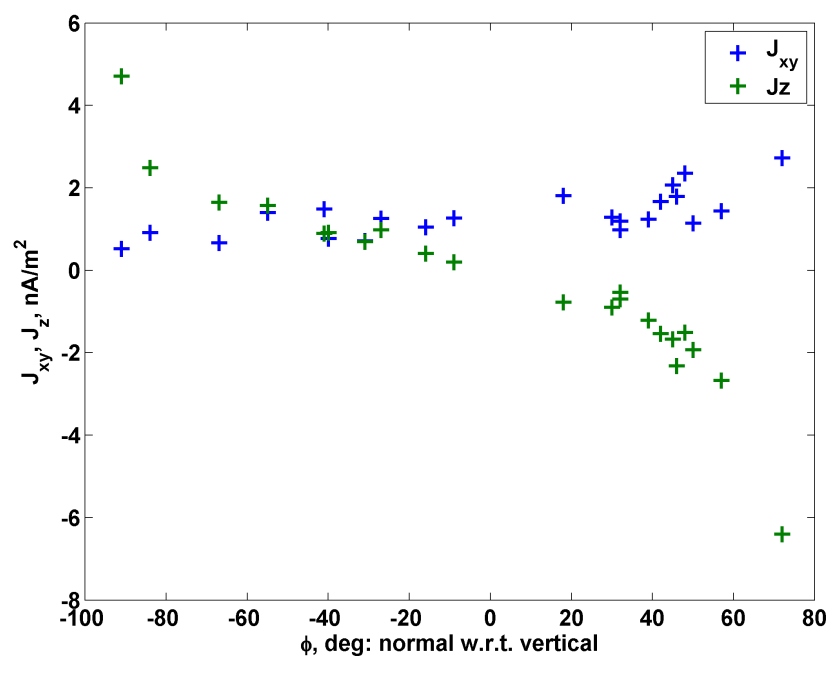

Fig. 7. $J_{x y}=\sqrt{J_{x}^{2}+J_{y}^{2}}$ and $J_{z}$ current density components relative to tilts for all individual crossings in Fig. 2.

is almost constant (close to Harris within the given range of relative magnetic field). Therefore this result also does not contradict our model. However, the difference between components is not so vivid, probably because not all crossings from this data set were individually identified with the slippage deformation.

\section{Discussion and conclusions}

The diversity of current sheet crossings observed by the Cluster spacecraft in the Earth's magnetotail, suggests variety of

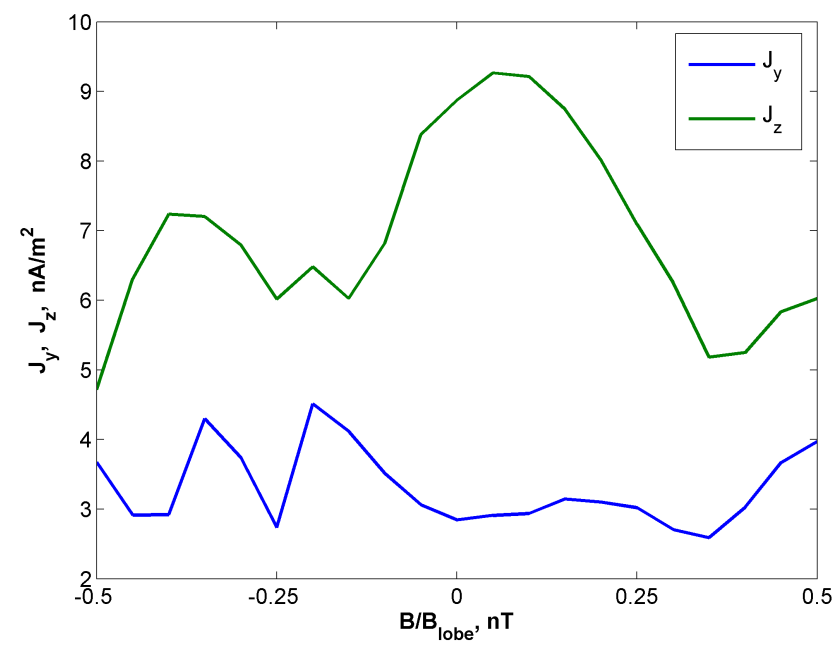

Fig. 8. Average current density profiles for 16 sheets from the Runov et al. (2006) dataset (see text for details).

physical driving mechanisms. We concentrate on a specific subset of flapping events: wavy crossings with alternating directions in the quiet plasma sheet with large $B_{z}$. The previously introduced by Petrukovich et al. (2006) slip deformation scheme supposes that the observed thin tilted sheets actually are the inner dynamic layer formed by relative (in the $Y$ coordinate) up-and-down motions of magnetic flux tubes (Fig. 1). This interpretation was mainly supported by the stability of the $B_{z}$ magnetic field component in neighboring crossings with almost opposite tilts. The $B_{y}$ component (after extraction of the flaring contribution coupled with $B_{x}$ ) remained small, so that flux tubes lie (almost) in a vertical plane in agreement with the proposed model geometry.

In this report we introduced a simple model of vertically moving flux tubes in an originally horizontal plasma sheet with a Harris profile. In the frame of this model tilting and thinning of the observed current sheet is strictly related to the appearance of a $J_{z}$ current density component, while $J_{y}$ remains equal to its nominal Harris value. In the data examples similar variability of $J_{z}$ was detected. The small relatively constant $J_{x}$ is most likely a part of the cross-tail current and is not in contradiction with this scheme. We thus conclude that the proposed slippage model of plasma sheet deformation is consistent with the current density observed within crossings. Thus many fast flapping events, being visually ideal objects for studying the current sheet, are actually dynamical deformations, rather than caused by fast coherent motion. Therefore some observed sheet profiles may be irrelevant to the quiet sheet structure.

It is also important that the combination of "elementary" slipping deformations (e.g. with different frequencies) in an initially Harris-type sheet may lead to observation of embedded, asymmetric and bifurcated current density profiles. In terms of this model an explanation of the observed variety 
of current density profiles is reduced to a proper selection of (irregular) slip deformations in each particular case. Since the required bulk plasma velocities are of the order of 10$50 \mathrm{kms}^{-1}$, the presence of such fluctuations in the plasma sheet is not improbable. A similar idea about origins of bifurcated profiles was earlier suggested by Hoshino et al. (1996).

Essentially a slip deformation (as well as any differential motion) is a violation of the sheet one-dimensionality and stationarity conditions, which are necessary for the interpretation of magnetic gradient measurements. In the case of a "nice-looking" flapping event with more or less coherent change of $B_{x}$, it seems reasonable to assume that the sheet is stationary and one-dimensional at least locally, and indeed the proper frame of reference is usually well defined. However, as is shown in our examples, even order of magnitude smaller and shorter-scale variations, visually preserving the integrity of a crossing, can substantially vary $J_{z}$ (and the local tilt). Since each flapping event is characterized by a single (averaged or fitted) normal, all these smaller-scale current density variations will be understood as the extrema on the current density profile. Of course, beyond our simple model, similar sources may act on $J_{y}$ and $J_{x}$, and an initial non-Harris structure of the sheet (e.g. Zelenyi et al., 2006) may also contribute. Summarizing, the approximation of a single normal for the whole crossing may work quite well as a general characteristic, but may be not sufficient to interpret the cross-sheet current density profiles.

Unfortunately, unambiguous differentiation between tilt variations and profile variations (non-linearity) is impossible with four spacecraft and three-dimensional space. Therefore to interpret the observed profiles as signatures of the real current sheet structure one has to use extra arguments, e.g. statistical analysis or placing limitations on the sheet tilt.

In conclusion, the slippage model of the formation of strongly tilted sheets in the magnetotail plasma sheet is further substantiated by comparison of the model and observed current density profiles. More measurement points than Cluster has now, are necessary to reliably distinguish between non-linear current sheet profiles and variations of the geometry of the sheet or its non-stationarity. This goal may be achieved by proper planning of the spacecraft separation in the Cross-Scale mission.

Acknowledgements. The work was supported in part by the RFBR grant 06-05-90631. A. A. P. would like to acknowledge the academic exchange program and hospitality of IWF.

Editor-in-Chief W. Kofman thanks S. Cowley and another anonymous referee for their help in evaluating this paper.

\section{References}

Asano, Y., Nakamura, R., Baumjohann, W., Runov, A., Vörös, Z., Volwerk, M., Zhang, T. L., Balogh, A., Klecker, B., and Rème, H.: How typical are atypical current sheets?, Geophys. Res. Lett, 32, L03108, doi:10.1029/2004GL021834, 2005.
Balogh, A., Carr, C. M., Acuña, M. H., et al.: The Cluster magnetic field investigation: overview of in-flight performance and initial results, Ann. Geophys., 19, 1207-1217, 2001, http://www.ann-geophys.net/19/1207/2001/.

Hoshino, M., Nishida, A., Mukai, T., Saito, Y., Yamamoto, T., and Kokubun, S.: Structure of plasma sheet in magnetotail: Doublepeaked electric current sheet, J. Geophys. Res., 101, $24775-$ 24786, 1996.

Lui, A. T. Y., Meng, C.-I., and Akasofu, S.-I.: Wavy nature of the magnetotail neutral sheet, Geophys. Res. Lett., 5, 279-282, 1978.

Petrukovich, A. A., Baumjohann, W., Nakamura, R., Balogh, A., Mukai, T., Glassmeier, K.-H., Rème, H., and Klecker, B.: Plasma sheet structure during strongly northward IMF, J. Geophys. Res., 108, 1258, doi:10.1029/2002JA009738, 2003.

Petrukovich, A. A., Baumjohann, W., Nakamura, R., Runov, A., and Balogh, A.: Cluster vision of the magnetotail current sheet on a macro-scale, J. Geophys. Res., 110, A06204, doi:10.1029/2004JA010825, 2005.

Petrukovich, A. A., Zhang, T. L., Baumjohann, W., Nakamura, R., Runov, A., Balogh, A., and Carr, C.: Oscillatory magnetic flux tube slippage in the plasma sheet, Ann. Geophys., 24, 16951704, 2006,

http://www.ann-geophys.net/24/1695/2006/.

Petrukovich, A. A., Baumjohann, W., Nakamura, R., Runov, A., Balogh, A., and Reme, H.: Thinning and stretching of the plasma sheet, J. Geophys. Res., 112, A10213, doi:10.1029/2007JA012349, 2007.

Runov, A., Nakamura, R., Baumjohann, W., Zhang, T. L., Volwerk, M., Eichelberger, H.-U., and Balogh, A.: Cluster observation of a bifurcated current sheet, Geophys. Res. Lett., 30, 1036, doi:10.1029/2002GL016136, 2003.

Runov, A., Sergeev, V., Nakamura, R., Baumjohann, W., Vörös, Z., Volwerk, M., Asano, Y., Klecker, B., Rème, H., and Balogh, A., Properties of a bifurcated current sheet observed on 29 August 2001, Ann. Geophys., 22, 2535-2540, 2004, http://www.ann-geophys.net/22/2535/2004/.

Runov, A., Sergeev, V.A., Baumjohann, W., Nakamura, R., Apatenkov, S., Asano, Y., Volwerk, M., Vörös, Z., Zhang, T. L., Petrukovich, A., Balogh, A., Sauvaud, J.-A., Klecker, B., and Rème, H.: Electric current and magnetic geometry in flapping magnetotail current sheets, Ann. Geophys., 23, 1391-1403, 2005a, http://www.ann-geophys.net/23/1391/2005/.

Runov, A., Sergeev, V. A., Nakamura, R., Baumjohann, W., Zhang, T. L., Asano, Y., Volwerk, M., Vörös, Z., Balogh, A., Sauvaud, J.-A., and Rème, H.: Reconstruction of the magnetotail current sheet structure using multi-point Cluster measurements, Planetary Space Sci., 53, 237-243, 2005b.

Runov, A., Sergeev, V. A., Nakamura, R., Baumjohann, W., Apatenkov, S., Asano, Y., Takada, T., Volwerk, M., Vörös, Z., Zhang, T. L., Sauvaud, J.-A., Rème, H., and Balogh, A.: Local structure of the magnetotail current sheet:(2001 Cluster observations, Ann. Geophys, 24, 247-262, 2006.

Sharma, A. S., Nakamura, R., Runov, A., et al: Transient and localized processes in the magnetotail: A review, Ann. Geophys., 26, 955-1006, 2008, http://www.ann-geophys.net/26/955/2008/.

Sergeev, V., Runov, A., Baumjohann, W., Nakamura, R., Zhang, T. 
L., Volwerk, M., Balogh, A., Rème, H., Sauvaud, J.-A., Andre, M., and Klecker, B.: Current sheet flapping motion and structure observed by Cluster, Geophys. Res. Lett., 30, 1327, doi:10.1029/2002GL016500, 2003.

Sergeev, V., Runov, A., Baumjohann, W., Nakamura, R., Zhang, T. L., Balogh, A., Louarn, P., Sauvaud, J.-A., and Rème, H.: Orientation and propagation of current sheet oscillations, Geophys. Res. Lett., 31, L05807, doi:10.1029/2003GL019346, 2004.

Thompson, S. M., Kivelson, M. G., El-Alaoui, M., Balogh, A., Rème, H., and Kistler, L. M.: Bifurcated current sheets: Statistics from Cluster magnetometer measurements, J. Geophys. Res., 111, A03212, doi:10.1029/2005JA011009, 2006.
Zelenyi, L. M., Malova, H. V., Popov, V. Y., Delcourt, D. C., Ganushkina, N. Y., and Sharma, A. S.: "Matreshka" model of multilayered current sheet, Geophys. Res. Lett., 33, L05105, doi:10.1029/2005GL025117, 2006.

Zhang, T. L., Baumjohann, W., Nakamura, R., Balogh, A., Glassmeier, K.-H.: A wavy twisted neutral sheet observed by Cluster, Geophys. Res. Lett., 29, 1899, doi:10.1029/2002/GL015544, 2002. 